\title{
Acute subdural and intratemporal hematoma as a complication of percutaneous compression of the gasserian ganglion for trigeminal neuralgia
}

\author{
I. Arrese; R.D. Lobato; J.F. Alén; A. Lagares and P. Miranda
}

Service of Neurosurgery. Hospital 12 de Octubre. Madrid.

\section{Summary}

The case of a 68 year-old man who developed a fatal intracranial hemorrhagic complication following percutaneous compression of the gasserian ganglion for trigeminal neuralgia is reported. The complication was likely related to improper placement of the Fogarty catheter into the temporal fossa out of the Mecke1,s cave. The anatomical structures at risk of damage by misplaced needle or catheter and some relevant thecnical details aimed to prevent extratrigeminal complications related with this and other percutaneous trigeminal lesioning procedures are analyzed.

KEY WORDS: trigeminal neuralgia. Gasserian ganglion. Intracerebral hemorrhage. Subdural hematoma. Percutaneous compression trigeminal ganglion.

Hematoma subdural e intraparenquimatoso agudo como complicación de la compresión percutánea del ganglio de Gasser

\section{Resumen}

Se presenta el caso de un paciente de 68 años de edad con neuralgia esencial del trigémino que fue operado mediante compresión percutanea del ganglio de Gasser y desarrolló una hemorragia intracraneal que resultó fatal. La complicación se relacionó probablemente con el emplazamiento erróneo del cateter de Fogarty y el inflado del balón fuera del cavum de Meckel. Se analiza la disposición de las estructuras anatómicas susceptibles de sufrir daño mecánico durante este procedimiento quirúrgico y se comentan algunos aspectos técnicos a tener en cuenta para prevenir el desarrollo de complicaciones como la ocurrida en nuestro paciente.

PALABRAS CLAVE: Neuralgia trigeminal. Ganglio de Gasser. Compresión percutanea del ganglio trigeminal.

Recibido: 16-08-04. Aceptado: 15-09-04
Hemorragia intracraneal. Hematoma subdural. Hematoma intracerebral.

\section{Introduction}

Percutaneous compression of the trigeminal ganglion (PCTG) is an effective and safe surgical technique for trigeminal neuralgia which is thought to be almost free of major complications $\mathrm{s}^{1-3,5,7,14-17,22}$. Apart from intratrigeminal side effects such as transient hemifacial sensory loss, dysesthesia and masticatory weakness, extratrigeminal complications including oculomotor nerve palsies, extracranial arteriovenous fistula or carotid cavernous fistula have been occasionally described. Though different types of intracranial bleeding have been reported in the largest series of patients undergoing radiofrequency (RF) lesioning of the gasserian ganglion ${ }^{26,27}$, to our knowledge this is the first report of focal intracranial hemorrhage complicating PCTG.

We describe the case of a patient who developed a fatal intratemporal and subdural hemorrhage following PCTG and discuss the possible causes of technical failure with the aim of preventing such a dreadful complication.

\section{Case report}

This 68 year-old normotensive man had been suffering from typical trigeminal neuralgia located at the second and third trigeminal divisions on the left side for 6 years. Four years before the last admission he underwent PCTG at our Unit with a good functional result; entry into the foramen ovale with a 14-gauge needle-cannula was readly achieved under lateral fluoroscopic control, a 4 French Fogarty catheter was placed into the Meckel,s cave and the balloon was inflated for one minute showing the typical pear shape. Following surgery he presented the characteristic hemifacial hypoesthesia and was pain free until 6 months before admission when he was readmitted for a new PCTG

Abbreviations used in this paper. PCTG: percutaneous compression of the trigeminal ganglion. RF: radiofrequency. 
because recurrent neuralgia unresponsive to medical therapy. Routine preoperative analyses, coagulation studies included, were normal. At the second operation the balloon was again inflated during one minute as the surgeon considered the shape to be appropriate. However, the exam of the x-ray obtained during balloon inflation showed a cylindrical shape revealing its location out of the Meckel, cave. (Fig. 1). Changes in arterial pressure were not recorded during the whole procedure. The patient showed a slow and incomplete recovery of the level of consciousness and five hours after surgery he became comatose. A CT scan showed and acute subdural hematoma of the basal and convexity left temporal regions, together with an anterior temporal lobe hematoma on the side of operation. There was a marked midline shift. (Fig. 2) The family of the patient rejected surgical intervention and the patient died few days later.

\section{Discussion}

Our own experience and that of other authors suggest that PCTG is the simplest and less risky percutaneous tech-

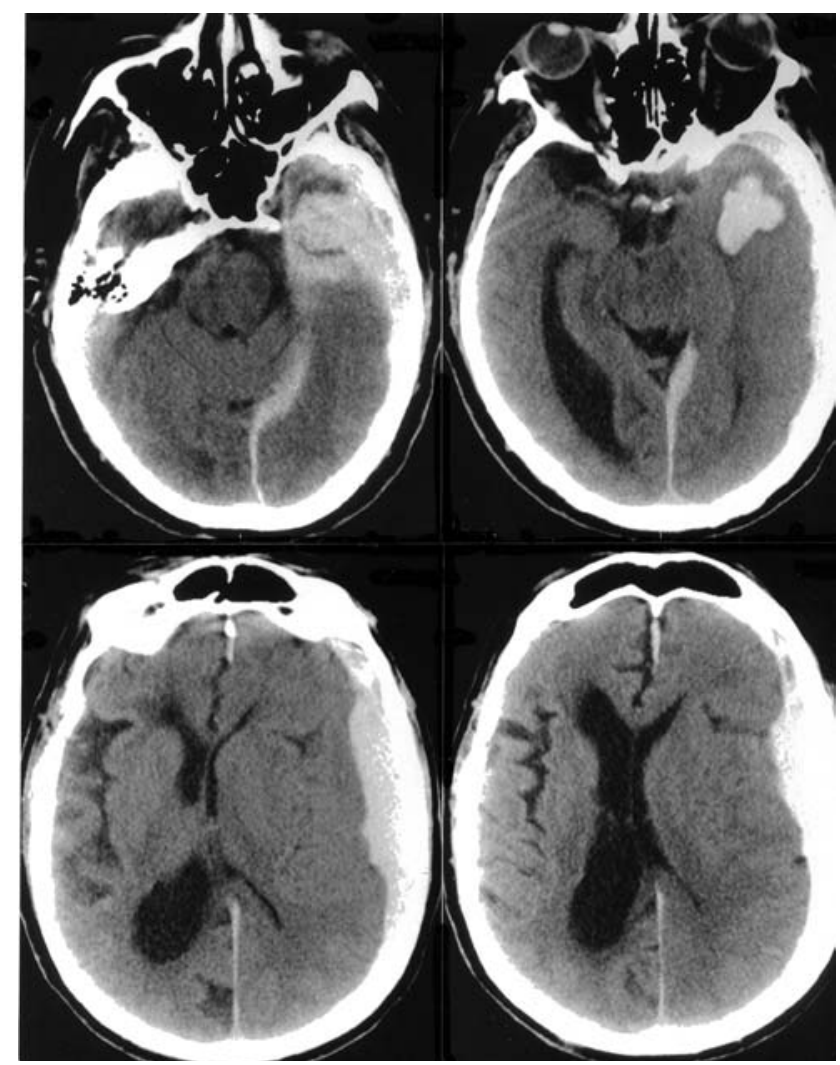

Figures 2. Postoperative CT scan performed five hours after surgery when the patient was comatose. A temporobasal, medial and convexity subdural hematoma together with an intratemporal clot is shown. There is uncal herniation and marked midline shift.
2005; 16:177-182

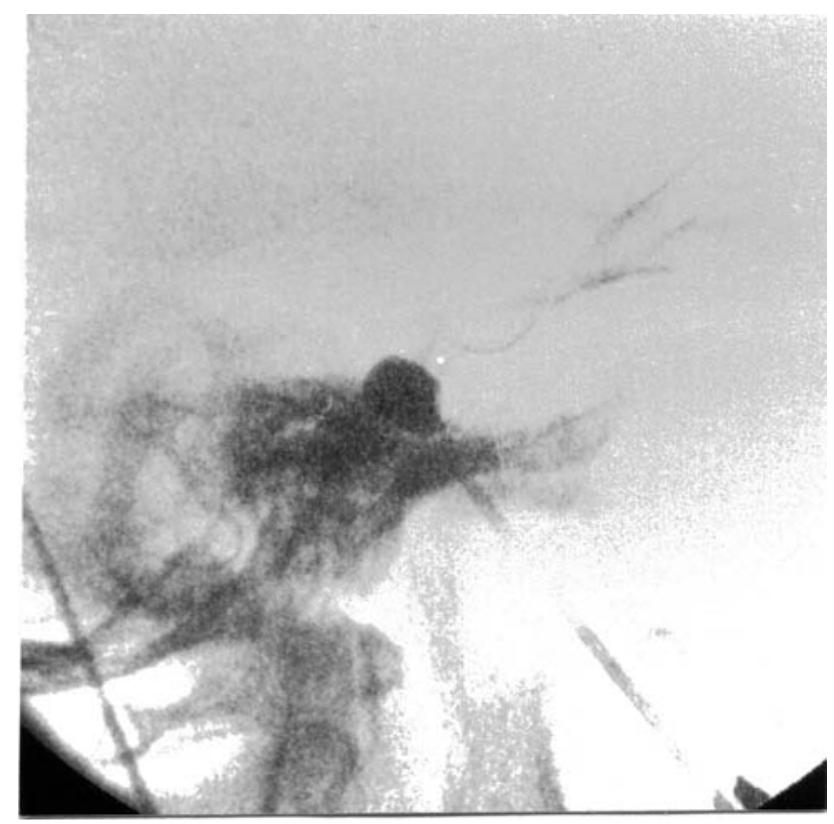

Figure 1. Fluoroscopic control of the position and shape of the inflated balloon during PCTG. The tip of the needle seems to be at the foramen ovale margins, but the balloon shows a cylindrical (like "in vitro") shape, it is not fully filled with contrast as air remained in its anterior part.

nique for treating trigeminal neuralgia, provided that both an improper placement of the needle-cannula or inflation of the balloon out of the Meckle,s cave are avoided ${ }^{1-3,5,7,14-}$ 17,22,29. Since during PCTG the peedle has not to be placed beyond the foramen ovale margins, one could expect the number of needle-related intracranial vascular, hemorrhagic or infectious complications to be lower than with radiofrequency (RF) lesioning or glycerol injection. In fact, when reviewing the literature extratrigeminal complications following PCTG seem less frequent than with other percutaneous techniques in which the peedle has to entry the trigeminal cistern ${ }^{1-3,5,9,11,14-17,22,25-28}$. However, it should be noted that the total number of patients treated with PCTG is also lower than those undergoing PF lesioning.

Only few patients treated with PCTG have been reported suffering extra or intracranial vascular or hemorrhagic complications. Gerber and Mullan ${ }^{7}$ reported two extracranial (pterygoid) arteriovenous fistulas in the region of the foramen ovale fed by the maxillary artery in one instance; the fistula caused tinnitus which resolved spontaneously in one case and required transarterial embolization in other. Reiwlta et al, ${ }^{18}$ reported a patient who developed an arteriovenous fistula in the region of the territory of the external carotid artery with the fistulous connection arising at the origin of the middle meningeal artery from the pterygopalatine artery which was punctured because of the posterolateral direction of the needle emerging from 
the foramen ovale; the clinical course was benign with spontaneous closure of the fistula 19 days after puncture. Kuether el al, ${ }^{13}$ reported the case of a 78 -year-old man who developed a direct high-flow carotid cavernous fistula which required endovascular treatment; the Fogarty catheter was apparently inflated into the lumen of the internal carotid artery despite that no arterial hemorrhage was noted during the procedure. We also observed a low-flow carotid-cavernous fistula in a patient in whom the Meckel,s cave could not be entered which resolved spontaneously in 3 months ${ }^{17}$; in this patient repeated needle insertions using different trajectories always resulted in brisk, pulsatile arterial bleeding, probably arising from the internal carotid artery. Spaziante el al, ${ }^{24}$ reported the occurrence of subarachnoid hemorrhage filling the basal and sylvian cisterns in a 62 year old man who underwent PCTG; normal pressure hydrocephalus developed as a consequence, and the patient eventually died following various complications; the surgical procedure was apparently correct as the needle did not penetrate beyond the foramen ovale and the inflated balloon did not move out of the Meckel,s cave; in addition, the functional result was excellent indicating an appropriate compression of the gasserian ganglion, and both normal coagulation studies and cerebral angiography excluded an alternative cause for SAH in this patient; since he did not show arterial pressure rises during the procedure, the authors attributed subarachnoid hemorrhage to piercing of the dura at the intracranial entry point with subsequent hemorrhagic extension into the CSF spaces. In a comment on this report, Apfelbaum ${ }^{24}$ reported a personal case of fatal intracerebral hemorrhage following RF lesioning in a patient in whom he was certain that the needle electrode had never exited out of the Meckel, s cave.

When reviewing the literature on the three percutaneous lesioning procedures of the gasserian ganglion relevant data emerge which must be considered for diminishing or preventing the risk of vascular or hemorrhagic intracranial complications. Concerning PCTG, which requires a comparatively large needle than RF lesioning or glycerol injection, it is critical not penetrate beyond the foramen ovale. However, in some cases the tip of the needle is mislocated intracranially as evidenciated by either X-ray control, or the exit of CSF following stylet withdrawal. It should noted in this respect that when the needle tip is at the porus trigemnni; it is 5 to $11 \mathrm{~mm}$ behind the profile of the clivus as seen in the lateral fluoroscopic control ${ }^{26}$, and that the exit of CSF does not necessarily means that the needle,s tip is into the cistern of the Meckels,s cave as it may flow from the subtemporal subarachnoid space.

When the neede pass the foramen ovale a too steep insertion trajectory may carry it too far upward into the subtemporal subarachnoid space or against the temporal lobe, and when it is too far posterior it may enter the bra- instem ${ }^{26}$. When it is placed too medial it may go directly into the cavernous sinus and the internal carotid artery may be punctured. This risk is higher in patients with a primitive foramen lacerum medius, which consists of fusion of the foramen ovale with the foramen lacerum ${ }^{21,23}$, in fact, the bone wall separating the carotid artery from the trigeminal nerve may be paper-thin, or even absent ${ }^{10}$. In addition, a tortuous carotid artery may pass directly over the foramen ovale making it impossible reaching the ganglion without puncturing the artery $^{30}$.

Provided that the needle is appropriately positioned into the foramen ovale, venous bleeding may originate from the venous plexus crossing the foramen margins, and arterial bleeding may arise either from the meningeal accessory artery traversing the foramen, or from other local branches of the meningeal arteries ${ }^{12,23}$. Venous bleeding is readly identified, and arterialized blood coming from sources other than the internal carotid artery is also recognizable because its low and non pulsatile flow. In our experience about $12 \%$ of the patients undergoing PCTG show some type of bleeding through the needle during the procedure, most commonly venous in origin, and it is our policy do not alter the course of the operation when these two types of venous or arterial low flow bleeding occur as there is not evidence that it may result in clinically relevant extra or intracranial hemorrhagic complications ${ }^{16,17}$.

Keeping the needle within the foramen ovale all through PCTG makes very unlikely that the Fogarty catheter which has a soft, blunt tip, perforates the dura propia of the Meckel,s cave, or the lumen of the carotid artery. However, even if the needle seems to be correctly placed, it is not impossible for the catheter reaching the temporal fossa out of the cave as we have observed a cylindrical or "in vitro" like appearance of the balloon in patients who had the needle,s tip at or immediately below the limit of the foramen ovale suggesting that dural piercing had occurred during needle,s insertion allowing the catheter to slip out of the cave. Needle repositioning because the inflated balloon does not show a "pear" shape occurs in about $14 \%$ of the cases in our practice, and it should be noted that in many instances of catheter misplacement the resistance felt to its advancement when it is pushed out of the needle,s lumen is similar to that encountered when it is pushed for entering the cave. Thus, despite a rigorous puncture technique and an apparently satisfactory $\mathrm{x}$-ray picture showing a correct relation of the needle with bony landmarks, the only manner to ascertain the appropriate location of the catheter is checking the shape of the inflated balloon; when the appearance is not the typical "péar-shaped" one, it is better immediately deflated and the needle repositioned. This policy was not followed in the patient reported here in whom the foramen ovale limit was likely trespassed with the needle leading to catheter mislocation and balloon inflation out of the cave. A 
necropsic study could not be performed in our patient, but it is our impression that Fogarty catheter slippage occurred somewhere intratemporally when it was pushed forward for entering the cave resulting in balloon inflation into the subarachnoid-subdural space or the temporal lobe itself.

Though it has been argued that an "in vitro" like, or cylindrical-shaped balloon may also be observed in patients with a large Meckle,s cave ${ }^{14,22}$, to us it reveals an erroneous location as we have been unable to advance the catheter into the posterior fossa through the poros trigemini following balloon,s deflation when such a shape is initially observed; in contrast, when the pear shape is observed the catheter may be pushed into the posterior fossa following balloon,s deflation whithout any resistance. We and other authors have observed postoperative transient oculomotor palsies when a balloon showing an "in vitro" like appearance is kept inflated more than the time necessary for checking its shape; however, the exact mechanism of oculomotor dysfunction in these cases remains to be determined. Postoperative transient diplopia occurring in patients showing a pear-shaped balloon during surgery and satisfactory functional result have been attributed to IV or VI nerve compression against the tentorium o within the cavernous sinus $^{14,17,29}$.

Occasionally we have observed another atypical balloon shapes such as oval, irregular or round which resulted in a good functional result as revealed by long-lasting pain control with the corresponding hemifacial hipoesthesia, despite that the nipple protruding through the poros trigemini and providing the typical pear-shape of a properly placed balloon was not observed. We indirectly assumed that the balloon was inside the Meckel,s cave in these instances, but we have not an explanation for these atypical shapes ${ }^{17}$.

Intracranial complications may also result when a needle is inserted through other cranial base holes few millimeters distant from the foramen ovale. Sweel and Polelli ${ }^{27}$ reported a left intratemporal hemorrhage in a patient undergoing RF lesioning after the needle entered the so called "foramen or canal of Vesalius" which is anterior and/or medial to the ovale and may be large enough to admit an 18-gauge needle. Another foramen close to the ovale is the innominate foramen or canaliculus of Arnold. Though it seems very difficult for the 14 needle gauge used in PCTG penetrating these small diameter cranial base holes, this risk may be prevented by careful fluoroscopic control resurting not only to the routine lateral projection, but to anteroposterior or submentovertex ones if necessary.

To diminish the risk of both extracranial (subpterygoid) and intracranial needle-related hemorrhagic complications in patients treated by PCTG, Gerher ${ }^{6}$ uses a modified $n^{\circ} 13$ Cone trochar and cannula; the skin is pierced with the shapp trochar which is advanced with the cannula until it reaches the proximity of the foramen ovale when it is replaced by a blunt trochar for engaging the foramen. It seems very likely that with this trochar the risk of piercing the dura propia of the cave actually decreases.

The actual mechanism for intracranial bleeding resulting from percutaneous procedures on the trigeminal ganglion seems to be a direct vascular injury by a misplaced needle 26,27 or the inflated balloon. The inferior temporal veins are divided into a lateral group which can not be reached by a misplaced needle crossing the foramen ovale, and a medial group formed by the uncal, anterior hippocampal and medial temporal veins, which empties into the middle segment of the basal vein as it courses along the medial edge of the temporal lobe ${ }^{20}$; it is plausible that one of these last bridging veins can be torn during percutaneous trigeminal lesioning. In our patient the combination of a subdural hematoma located not only at the temporal convexity, but also in the basal and medial parts of the temporal fossa, together with the anterobasal intratemporal hematoma suggest that they resulted from bridging vein and parenchymal dysruption caused by the needle or a misplaced balloon.

Overall, the risk of intracranial complications related to the most commonly performed trigeminal lesioning procedures is low, and serious extratrigeminal adverse events were absent in centers operating over 1000 patients $^{27}$.

Excluding the patient reponed here, and some complaining from postoperative transient headache and showing discrete meningeal sings suggestive of mild subsrachnoid hemorrhage, we have not had intracranial hemorrhagic complications in more than 1000 percutaneous retrogasserian trigeminal procedures (about two thirds RF lesions and one third PCTG). Sweet and Poletti ${ }^{27}$ made a survey of complications of percutaneous RF trigeminal rhizotomies and recruited data from 29 neurosurgical units totalling over 7000 cases, mostly undergoing RF lesioning. When analyzing extratrigeminal complications they found that 5 patients developed a carotid-cavernous fistula and 18 oculomotor palsies. Focal intracranial hemorrhages occurred in 19 patients. In one case an infratemporal subdural hematoma occurred because of a needle puncture of the inferior temporal vein, the clot was removed and the patient recovered. Three patients had hemorrhages of the ipsilateral intratemporal lobea two of these died and one was disabled. The remaining 15 intracerebral hemorrhages occurred at a site unrelated with the needle; eight of these patients died, and 4 developed severe disability. Apart from hemorrhagic strokes, other vascular accidenta related to the electrode were one arterial puncture followed by transient hemiparesis and 5 arterial subsrachnoid hemorrhages; three out of these last patients died and two recovered. In another patient showing brisk bleeding at the puncture site the operation was continued and the trigeminal lesion made after 
bleeding stopped spontaneously; six hours later a massive SAH ensued in the posterior and middle fossa bilaterally. Consequently, Sweet recommended interrupting RF rhizotomy when arterial bleeding is observed, even if its origin is the extracranial carotid artery, and resuming it some days later when the puncture site is healed ${ }^{26,27}$. As stated above we never stopped PCTG because the occurrence of arterial bleeding through the needle, even if suggestive of carotid origin as it invariably disappeared following needle replacement excepting in the patient in whom repeated puncture of the foramen ovale always resulted in brisk and pulsatile arterial bleeding most likely arising from the carotid artery.

Sweel ${ }^{25}$, has emphasized that intracranial hemorrhages occurring in patients exposed to percutaneous trigeminal lesioning procedures are not always puncture-related, but can be attributed to an anticoagulant or bleeding tendency, or to abrupt arterial blood pressure rises occurring during surgery. Consequently, he has recommended performing careful preoperative coagulation studies as most patients suffering trigeminal neuralgia are old and many are on aspirin and other drugs, carbamazepine among them, which are able to increase bleeding risk. On the other hand, a close observation and careful control of the arterial pressure changes during the operation is mandatory, as many patients develop sudden rises in blood pressure which may also result in intracerebral hemorrhage or ischemic cardiac complications ${ }^{25}$. The mechanism of blood pressure elevation during gasserian ganglion heating is unknown, but it has been related with pain felt by the unanesthetized patient during the procedure. We have also observed marked rises in arterial blood pressure during compression of the gasserian ganglion even under general anesthesia, which may be easily controlled with nitroprusside or better prevented by injecting lidocaine into the Meckel,s cave before inflating the balloon ${ }^{4,16,17}$. In the patient reported here there were not changes in arterial pressure coincidental with balloon inflation, likely because it was out of the cave.

To conclude, we have reported the first case of a fatal intracranial hemorrhagic complication of PCTG. The most likely explanation for bleding was Fogarty catheter slippage and prolonged inflation of the balloon out of Meckel,s cave due to failure of the surgeon to recognize its atypical shape. The mechanism could also be puncture related, but in any case, needle misplacement beyond or out of the foramen ovale initially during insertion is just another technical error which must be also avoided. The normal coagulation studies, the absence of arterial pressure changes during balloon inflation and the location of hematomas close to the surgical field make almost certain that bleeding was related to a local vascular injury in our patient.

\section{References}

1. Abdennebi, B., Bouatta, F., Chitti, M., Bougatene, B.: Percutaneous balloon compression of the gasserian ganglion in trigeminal neuralgia. Long term results in 150 cases. Acta Neurochir (Wien) 1995; 136: 72-74.

2. Belber, C.J., Rak, R.A.: Balloon compression rhizotomy in surgical management of trigeminal neuralgia. Neurosurgery 1987; 20: 908-913.

3. Brown, J.A., Mullan, S.: Percutaneous trigeminal nerve compression. In Neurosurgical Techniques. ( ${ }^{\text {rd }}$ ed) Vol 2 Scmideck HH (ed). WB Saunders Co, Philadelphia, 1995; pp : 1537-1542.

4. Domínguez, J., Lobato, R.D., Rivas, J.J. et al.: Changes in the systemic blood pressure and the cardiac rhythm induced by therapeutic compression of the trigeminal ganglion. Neurosurgery 1994; 34: 422-428.

5. Fraioli, B., Esposito, V., Guidetti, B., Cruccu, G., Manfredi, M.: Treatment of trigeminal neuralgia by thermocoagulation, glycerolization and percutaneous compression of the gasserain ganglion and/or retrogasserian rootlets; long term results and therapeutic protocol. Neurosurgery 1989; 24: 239245.

6. Gerber, A.U.: Needle for use during percutaneous compression of gasserian ganglia for trigeminal neuralgia. Technical note. J Neurosurg 1989; 21: 45.

7. Gerber, A.U., Mullan, S.F.: Trigeminal nerve compression for neuralgia. En Textbook of Stereotactic and Functional Neurosurgery. Gildenberg PL, Tasker KR (eds). McGraw Hill, New York, 1998; pp: 1707-1713.

8. Gokalp, H.Z., Kanpolat, Y., Tumer, B.: Carotid-cavernous fistula following percutaneous trigeminal ganglion approach. Clin Neurol Neurosurg 1980; 82: 269-272.

9. Hakanson, S.: Transoval trigeminal cisternography. Surg Neurol 1978; 10: 137-144.

10. Harris, F.S., Rhoton, A.L. Jr.: Anatomy of the cavernous sinus. A microsurgical study. J Neurosurg 1976; 45:169180 .

11. Horwitz, N.H., Rizzoli, H.V.: Postoperative complications of intracranial neurological surgery. Baltimore, Williams \& Wilkins, 1982.

12. Henderson, W.R.: The anatomy of the gasserian ganglion and the distribution of pain in relation to injections and operations for trigeminal neuralgia. Ann R Coll Surg Engl 1965; 37: 346-373.

13. Kuether, T.A., O,Neill, O.R., Nesbit, G.M., Barnwell, S.L.: Direct carotid cavernous fistula after trigeminal balloon microcompression gangliolysis: Case report. Neurosurgery 1996; 39: 853-856.

14. Lichtor, T., Mullan, J.F,: A 10-year follow-up review of percutaneous microcompression of the trigeminal ganglion. $\mathrm{J}$ Neurosurg 1990; 72: 49-54.

15. Mullan, J.F., Lichtor, T.: Percutaneous microcompres- 
sion of the trigeminal ganglion for trigeminal neuralgia. J Neurosurg 1983; 59: 1007-1012.

16. Lobato, R.D.: Percutaneous compression of the trigeminal ganglion for trigeminal neuralgia. En Treatment of Chronic pain Possibilities, Limitations and long-term followup, Mumenthaler M et al (eds). Harwood Academic Publishers, London, 1990; pp: 215-230.

17. Lobato, R.D., Rivas, J.J., Sarabia, R., Lamas, E.: Percutaneous microcompression of the gasserian ganglion for trigeminal neuralgia. J Neurosurg 1990; 72: 546-553.

18. Revuelta, R., Nathal, E., Balderrama, J., Tello, A., Zenteno, M.: External carotid artery fistula due to microcompression of the gasserian ganglion for relief of trigeminal neuralgia. J Neurosurg 1993; 78: 499-500.

20. Rhoton, A.L.: Cranial anatomy and surgical approaches. Lippincott Williams \& Wilkins, Schaumburg, 2003.

21. Sekhar, L.N., Heros, R.C., Kerber, C.W.: Carotidcavernous fistula following percutaneous retrogasserian procedures. Report of two cases. J Neurosurg 1979; 51:700-706

22. Skirving, D.J., Dan, N.G.: A 20-year review of percutaneous balloon compression of the trigeminal ganglion. $\mathrm{J}$ Neurosurg 2001; 94: 913-917.

23. Sondheimer, F.K.: Basal foramina and canals. In Newton TH, Potts DG (eds). Radiology of the Skull and Brain. Vol. 1 Book 1. St Louis: CV Mosby, 1971; pp: 287-347.

24. Spaziante, R., Cappabianca, P., Peca, C., Divitiis, E.: Subarachnoid hemorrhage and "normal pressure hydrocephalus". Fatal complication of percutaneous microcompression of the gasserian ganglion. Neurosurgery 1988; 22:148-151.

25. Sweet, W.H.: Dangerous rises in blood pressure upon heating of trigeminal rootlets; increased bleeding times in patients with trigeminal neuralgia. Neurosurgery 1985; 17 : 843-844.

26. Sweet, W.H.: Faciocephalic pain. En Brain Surgery. Appuzo MCJ (ed) Complications Avoidance and Management. Churchill Livingstone, New York 1993; pp: 2053-2083

27. Sweet, W.H., Poletti, C.E.: Complications of percutaneous rhizotomy and microvascular decompression operations for facial pain. En Neurosurgical Techniques. (3 $3^{\text {rd }}$ ed) Vol 2 Schmideck HH (ed). WB Saunders Co, Philadelphia, 1995; pp : 1687-1696.

28. Taha, J.M., Tew, J.M.: Radiofrequency rhizotomy for trigeminal and other cranial neuralgias. Gildenberg PL, Tasker RR (eds). McGraw Hill, New York, 1998, pp: 1707-1713

29. Urculo, E., Alfaro, R., Arrazola, M., Astudillo, E., Rejas, G.: Trochlear nerve palsy after repeated percutaneous balloon compression for recurrent trigeminal neuralgia: Case report and pathogenic considerations. Neurosurgery 2004; 54 : 505-509.

30. Wepsic, J.G.: Complications of percutaneous surgery for pain. Clin Neurosurg 1976; 23: 454-464.

Arrese, I.; Lobato, R.D.; Alén, J.F.; Lagares, A.; Miranda, P.: Acute subdural and intratemporal hematoma as a complication of percutaneous compression of the gasserian ganglion for trigeminal neuralgia. Neurocirugía 2005; 16 : $177-182$.

Correspondence: I Arrese. Servicio Neurocirugía. Hospital "12 de Octubre". Avda Cordoba s/n. 28041 Madrid. Spain. 